\title{
BMJ Open What treatments work for anxiety and depression in children and adolescents with chronic fatigue syndrome? An updated systematic review
}

\author{
Philippa Clery (D , ${ }^{1}$ Alexander Royston, ${ }^{1}$ Katie Driver, ${ }^{1}$ Jasmine Bailey, ${ }^{1}$ \\ Esther Crawley, ${ }^{1,2}$ Maria Loades ${ }^{1,3}$
}

To cite: Clery P, Royston A, Driver K, et al. What treatments work for anxiety and depression in children and adolescents with chronic fatigue syndrome? An updated systematic review. BMJ Open 2022;12:e051358. doi:10.1136/ bmjopen-2021-051358

- Prepublication history and additional supplemental material for this paper are available online. To view these files, please visit the journal online (http://dx.doi.org/10.1136/ bmjopen-2021-051358).

Received 18 March 2021 Accepted 03 December 2021

Check for updates

(c) Author(s) (or their employer(s)) 2022. Re-use permitted under CC BY. Published by BMJ.

${ }^{1}$ Centre for Academic Child Health, University of Bristol, Bristol, UK

${ }^{2}$ Paediatric Chronic Fatigue Syndrome Specialist Service, Royal United Hospitals Bath NHS Foundation Trust, Bath, UK ${ }^{3}$ Department of Psychology, University of Bath, Bath, UK

Correspondence to

Dr Philippa Clery;

philippa.clery@bristol.ac.uk

\section{ABSTRACT}

Objectives Children with chronic fatigue syndrome/ myalgic encephalomyelitis (CFS/ME) experience a higher prevalence of depression and anxiety compared with age-matched controls. Our previous systematic reviews in 2015/16 found little evidence for effective treatment for children with CFS/ME with comorbid depression and/or anxiety. This review updates these findings.

Design A systematic review. We searched Cochrane library, Medline, Embase and PsycINF0 databases from 2015 to 2020. We combined the updated results with our previous reviews in a narrative synthesis.

Participants Inclusion criteria: $<18$ years old; diagnosed with CFS/ME (using Centers for Disease Control and Prevention, National Institute for Health and Care Excellence or Oxford criteria); validated measures of depression and/or anxiety.

Interventions Observational studies or randomised controlled trials.

Comparison Any or none.

Outcomes Studies with outcome measures of anxiety, depression or fatigue.

Results The updated review identified two studies. This brings the total number of paediatric CFS/ME studies with a measure of anxiety and/or depression since 1991 to 16. None of the studies specifically targeted depression, nor anxiety. One new study showed the Lightning Process (in addition to specialist care) was more effective at reducing depressive and anxiety symptoms compared with specialist care alone. Previous studies evaluated cognitive-behavioural therapy (CBT); pharmacological interventions and behavioural approaches. CBT-type interventions had most evidence for improving comorbid anxiety and/or depressive symptoms but varied in delivery and modality. Other interventions showed promise but studies were small and have not been replicated. Conclusion Very few paediatric CFS/ME intervention studies have been conducted. This review update does not significantly add to what is known from previous reviews. The evidence is of poor quality and insufficient to conclude which interventions are effective at treating comorbid anxiety and/or depression in paediatric CFS/ME. PROSPERO registration numbers CRD42016043488 and CRD42015016813.
Strengths and limitations of this study

This review used a systematic approach to identify updated evidence for treatment approaches for comorbid anxiety and/or depression in paediatric chronic fatigue syndrome/myalgic encephalomyelitis, and combined it with previous review results to provide a comprehensive synthesis of all evidence available since 1991.

- Non-English language articles were included.

- Authors were contacted and subgroup data obtained when available.

- Grey literature and unpublished material was not included.

- There was insufficient data to carry out a meta-analysis.

\section{INTRODUCTION}

Chronic fatigue syndrome (CFS)/ myalgic encephalomyelitis (ME) is a common but poorly understood condition causing disabling fatigue, malaise, myalgia, sleep difficulties and problems concentrating. ${ }^{1}$ In children and adolescents (henceforth referred to as children), prevalence is estimated at $0.55 \%$ (95\% CI $0.22 \%$ to $1.35 \%$ ) across community, primary care and hospital populations. ${ }^{2} \mathrm{CFS} /$ ME has long-term impacts on children's physical, cognitive, emotional and social functioning. ${ }^{34}$

Children with CFS/ME suffer from higher rates of both depression and anxiety than agematched population samples. The prevalence estimates of comorbid depression and anxiety are $20 \%{ }^{5}$ and $29 \%,{ }^{6}$ respectively, compared with $2.1 \%$ and $7.2 \%^{7}$ in adolescents without CFS/ME. In those attending a specialist CFS/ ME service, $61 \%$ who meet diagnostic criteria for depression also have an anxiety disorder. ${ }^{5}$ Having comorbid depression and/or anxiety is associated with less favourable outcomes and may impact on engaging with treatment. 
Comorbid depression in paediatric CFS/ME is associated with greater functional disability, worse fatigue and more pain compared with those without depression. ${ }^{8}{ }^{9}$ Low mood, anergia and anhedonia could be barriers to motivation to engage in behavioural treatment approaches and cognitive behavioural therapy for fatigue (CBT-f). Depressive symptoms are therefore likely to require tailored treatment. ${ }^{9}$ The impact of anxiety on outcomes is less clear. Given that most children with CFS/ME who have anxiety also have depression, ${ }^{5}$ it is important to explore treatments for both.

Despite the high prevalence of comorbid mental health problems, there is little evidence about the effectiveness of treatments. Our two previous systematic reviews looking at depression and anxiety outcomes in existing CFS/ME intervention studies found that no specifically adapted treatments had been trialled to target depression and anxiety in paediatric CFS/ME. ${ }^{10}{ }^{11}$ Although CBT-f and a multicomponent inpatient programme showed promise in reducing depressive ${ }^{10}$ and anxiety ${ }^{11}$ symptoms, there was no consistent treatment approach for children with CFS/ME and comorbid depression or anxiety. Since conducting these reviews in 2015/2016, further intervention studies may have been published. It is important and timely to review the current evidence to provide an update on what treatments should be offered to this population. Further, it is important to consider anxiety and depression together given their overlap, whereas our previous reviews considered them separately.

We conducted an updated systematic review by synthesising the evidence regarding treatments for paediatric $\mathrm{CFS} / \mathrm{ME}$ and comorbid depression and anxiety since 2015. We combined these findings with results from our previous systematic reviews (1991-2015) to give an overview of all interventions evaluated since 1991 (when CFS/ ME was scientifically defined). Specifically, we aimed to address the following:

1. What treatment approaches are there for depression and anxiety in children with CFS/ME?

2. What is known about the treatment efficacy of these approaches for treating depression and anxiety in CFS/ME? Do different approaches have different outcomes?

\section{METHODS}

\section{Data sources and search strategy}

We conducted searches on Medline, Embase, PsycINFO and Cochrane Library databases. Searches were designed with input from an information specialist to include the concepts: paediatric; CFS/ME; anxiety and depression (search strategies are in online supplemental material). We updated the searches from when they had last been run (February 2015 for depression search; July 2016 for anxiety search) up until September 2020. The two searches were carried out by different reviewer teams: anxiety search (PC and $\mathrm{AR}$ ); depression search (KD and

\begin{tabular}{|c|c|c|}
\hline & Anxiety review & Depression review \\
\hline Participants & \multicolumn{2}{|c|}{$\begin{array}{l}\text { 1. Children }<18 \text { years of age } \\
\text { 2. Diagnosed with CFS/ME defined using one of } \\
\text { these criteria: } \\
\text { CDC aka Fukuda et } a l^{50} \\
\text { NICE }{ }^{1} \\
\text { Oxford aka Sharpe et } a l^{51}\end{array}$} \\
\hline Interventions & \multicolumn{2}{|c|}{$\begin{array}{l}\text { Observational cohort studies } \\
\text { Any study with intervention-for example, } \\
\text { observational clinical cohorts, clinical trials. }\end{array}$} \\
\hline Baseline measure & $\begin{array}{l}\text { Validated } \\
\text { assessment of } \\
\text { anxiety }\end{array}$ & $\begin{array}{l}\text { Validated assessment of } \\
\text { depression }\end{array}$ \\
\hline $\begin{array}{l}\text { Outcome } \\
\text { measure }\end{array}$ & $\begin{array}{l}\text { Either an anxiety } \\
\text { and/or fatigue } \\
\text { measure on } \\
\text { psychometrically } \\
\text { validated } \\
\text { assessments or } \\
\text { validated diagnostic } \\
\text { interviews. }\end{array}$ & $\begin{array}{l}\text { Either a depression and/ } \\
\text { or fatigue measure on } \\
\text { psychometrically validated } \\
\text { assessments or validated } \\
\text { diagnostic interviews. }\end{array}$ \\
\hline Language & \multicolumn{2}{|c|}{$\begin{array}{l}\text { Non-English language papers were considered } \\
\text { for inclusion. }\end{array}$} \\
\hline
\end{tabular}

CDC, Centers for Disease Control and Prevention; CFS/ME, chronic fatigue syndrome/myalgic encephalomyelitis; NICE, National Institute for Health and Care Excellence.

JB). Grey literature was not searched. Reference lists of articles for full-text screening were hand-searched.

\section{Inclusion and exclusion criteria}

Studies were included if they met inclusion criteria (table 1).

\section{Study selection}

Articles returned from database searches were inputted into Endnote and duplicates removed. Each reviewer (PC, $\mathrm{AR}, \mathrm{KD}, \mathrm{JB}$ ) conducted title and abstract screening independently. Full texts of potentially eligible articles were screened against specifically created eligibility checklists. The final articles for inclusion were cross-checked between all four reviewers and any conflicts discussed and resolved with input from the senior author (ML) if necessary. Where information from the paper was insufficient to determine eligibility, authors were contacted by email for additional information. If authors did not reply after two follow-up emails, the study was excluded. Figure 1 presents the PRISMA ${ }^{12}$ flow chart.

\section{Data extraction}

For all included articles, data were extracted independently by two reviewers (PC and AR) using a purposedesigned data extraction form to collect information about: study design; setting; recruitment; participant characteristics; CFS/ME definition used for diagnosis; assessment of depression and anxiety; other outcomes; treatment and interventions provided; definition of response and treatment/intervention outcomes. 


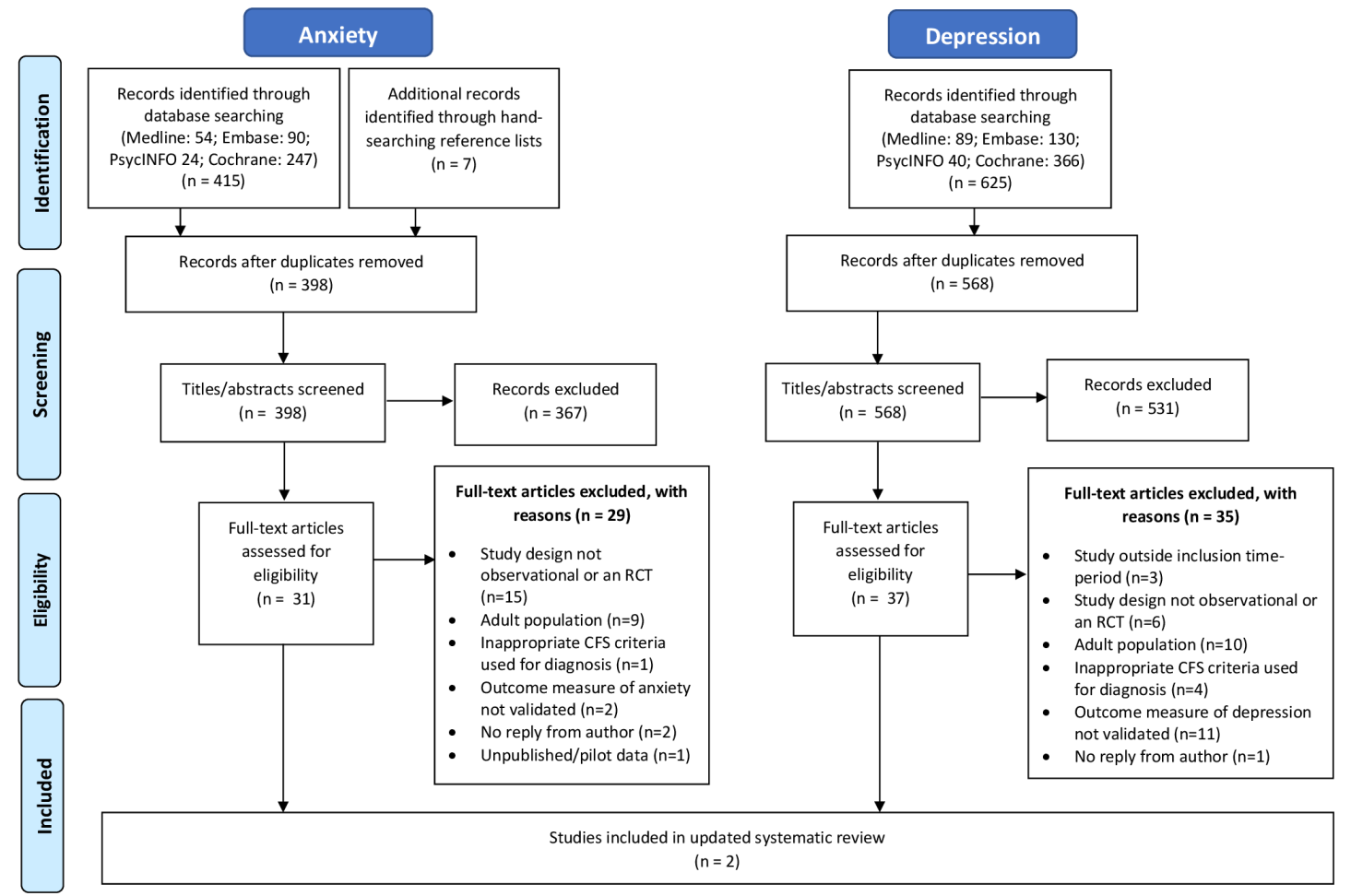

Figure 1 Flow chart for studies included in the systematic review; based on PRISMA guidelines. CFS, chronic fatigue syndrome; PRISMA, Preferred Reporting Items for Systematic Reviews and Meta-Analyses; RCT, randomised controlled trials.

\section{Quality assessment}

PC and AR used Risk of Bias (ROB) assessment tools ${ }^{13} 14$ to assess methodological quality of the included studies.

\section{Data synthesis}

We combined results from the included studies identified in the updated search with findings from the two previous systematic reviews ${ }^{10}{ }^{11}$ to conduct a narrative synthesis, ${ }^{15}$ providing an overview of all longitudinal studies that have been evaluated in this clinical cohort since 1991 (when CFS/ME was scientifically defined). There was insufficient comparable data to conduct a meta-analysis as interventions were heterogeneous and a range of outcome measures were reported. For each of the new studies, the effects of interventions on outcomes using mean differences were compared.

\section{Patient and public involvement}

No patients were involved.

\section{RESULTS}

\section{Studies included}

In the updated search (2015-2020), a total of 625 and 415 references were found by database searching for the depression and anxiety searches, respectively. After full-text screening, both searches returned the same two eligible studies. ${ }^{16}{ }^{17}$ One was a randomised controlled trial (RCT) ${ }^{17}$ one was a retrospective observational cohort study. ${ }^{16}$ The PRISMA ${ }^{12}$ flow chart is in figure 1 .

The previous systematic reviews for depression ${ }^{10}$ (search conducted in 2015) and anxiety ${ }^{11}$ (search conducted in 2016) found 362 and 1274 references, respectively. After full-text screening, the depression search returned nine eligible studies (one RCT, ${ }^{18}$ and eight observational studies $\left.{ }^{19-26}\right)$, and the anxiety search returned nine eligible papers from eight studies (three RCTs, ${ }^{27-30}$ six observational studies ${ }^{192122253132}$ ). Four of the studies from these two searches were the same.

Therefore, in total, 16 eligible studies were included in this narrative synthesis review. Figure 2 shows a flow chart combining studies from this updated search with studies identified from previous reviews.

\section{Quality assessment}

Of the total 16 studies in this review, 10 were observational and six were RCTs. Of the observational studies, five had an overall ROB as 'unclear', and five had 'high' ROB (as defined by the Cochrane ROB scale, ROBINS-I (Risk of Bias In Non-Randomised Studies) ${ }^{13}$ ). Of the RCTs, all six had an overall rating of 'low' ROB (as defined by the Cochrane ROB-2 scale). ${ }^{14}$ See online supplemental material for the quality assessment table. For detailed reporting on the quality assessment of studies from the previous searches, please refer to our previous two reviews. ${ }^{10} 11$

In this paper, we report in detail on the quality assessment of the two new studies found in the updated search.

The RCT ${ }^{17}$ was conducted by members of our CFS/ME research team (EC). The study has a low ROB from the concealed allocation randomisation process, minimal deviation from how interventions were intended to be delivered, and appropriate intention-to-treat analysis. Outcome measurement is biased because of self-reported 

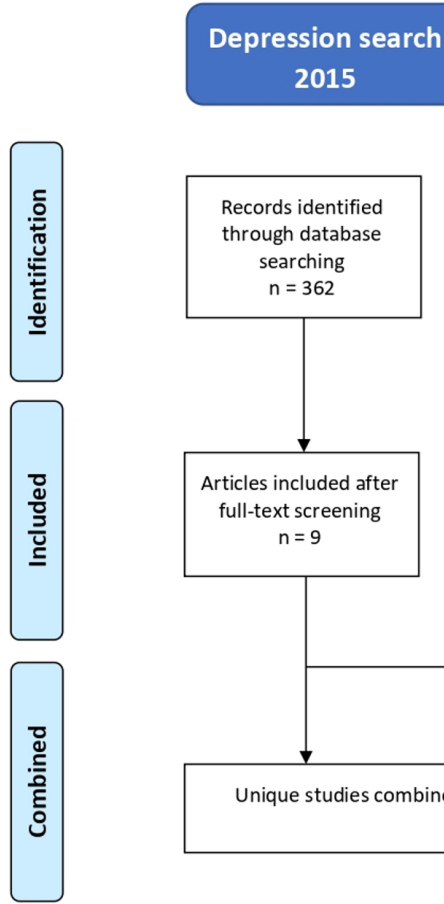

2015

Anxiety search

2016

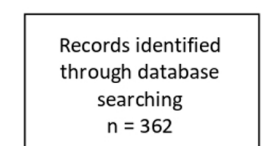

$n=362$

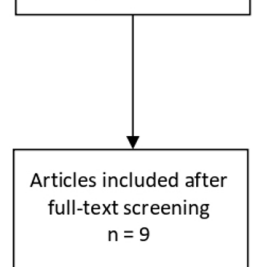

$n=9$
Records identified

through database searching $\mathrm{n}=1274$

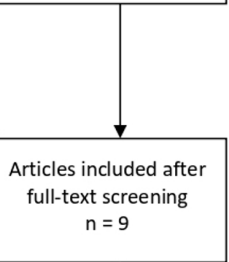

Updated Anxiety \& Depression search

Figure 2 Flow chart of studies combined from updated review and previous reviews.

measures, but this is standard for behavioural treatments. It is also biased due to loss to follow-up. In the control arm at 3 months, 13 of 49 (27\%) were lost to follow-up and at the primary outcome of 6 months, 12 of 49 (24\%) were not included in analysis. In the intervention arm 8 of $51(16 \%)$ were lost to follow-up at 3 months and 7 of $51(14 \%)$ were not included in primary analysis at 6 months. Although baseline characteristics between those who did and did not provide primary outcome data were similar, it is possible that missingness was related to the outcome.

The retrospective observational study ${ }^{16}$ is also biased due to poor follow-up rates at any one time point (making comparison difficult), and no pre-published analysis plan. In the cohort, there are two samples; one with baseline data for anxiety and depression and one without. Follow-up questionnaires were mailed to all participants on a number of occasions between January 2008 and June 2011. This produced a range of follow-up time points (1-21 years) after illness onset, meaning some patients would not have had contact with the clinic for a long time when they were sent the questionnaire, so it is likely that both disease status and time since illness influenced outcome data. Of the 489 patients who were sent baseline questionnaires, $74 \%$ returned a follow-up questionnaire on at least one occasion (range 1-7). For the sample of 366 without baseline data for anxiety and depression, $76 \%$ returned a follow-up questionnaire on one occasion, while only $8 \%$ returned a questionnaire on more than one occasion. Outcome measures were also self-reported, and many participants did not complete all measures.

\section{Participant and study characteristics}

The two studies identified in the updated search were: an RCT evaluating the 'Lightning Process' intervention alongside 'specialist medical care' compared with 'specialist medical care' alone ${ }^{17}$; and an observational cohort study assessing 'routine specialist care' over a 20 -year period. ${ }^{16}$ Studies from the previous reviews included the following. Four RCTs evaluating: inpatient programmes with predominantly behavioural approaches, ${ }^{18} 28$ an online CBT programme ${ }^{29} 30$ and intravenous gammaglobulin ${ }^{27}$; eight observational cohort studies evaluating: CBT, ${ }^{19} 2532$ CBT with pharmacotherapy, ${ }^{24} 31$ an antiviral treatment ${ }^{26}$ and an inpatient programme ${ }^{23}$; and two prospective observational community studies that did not assess a specified intervention. ${ }^{21} 22$ Follow-up times varied from immediately post-treatment to 21 years. Total number of participants included across all studies was 965. Most sample sizes were small but ranged between one and 418 . Participant ages ranged between 11 and 18. Most studies were conducted across Europe (UK, Netherlands, Spain) and Australia. One was in Japan, one in the USA (table 2).

None of the studies identified were specifically aimed at treating anxiety or depression in children with CFS/ ME (all primary outcomes were measures of fatigue or recovery). Anxiety and/or depression were measured as secondary outcomes using a variety of self-report questionnaires including the Hospital Anxiety and Depression Scale (HADS), ${ }^{33}$ Spence Children's Anxiety Scale (SCAS), ${ }^{34}$ the State-Trait Anxiety Inventory for Children ${ }^{35}$ the Multidimensional Anxiety Scale for Children, ${ }^{36}$ Spielberger State Trait Anxiety Questionnaire, ${ }^{37}$ Beck Depression Inventory, ${ }^{38}$ Children's Depression 


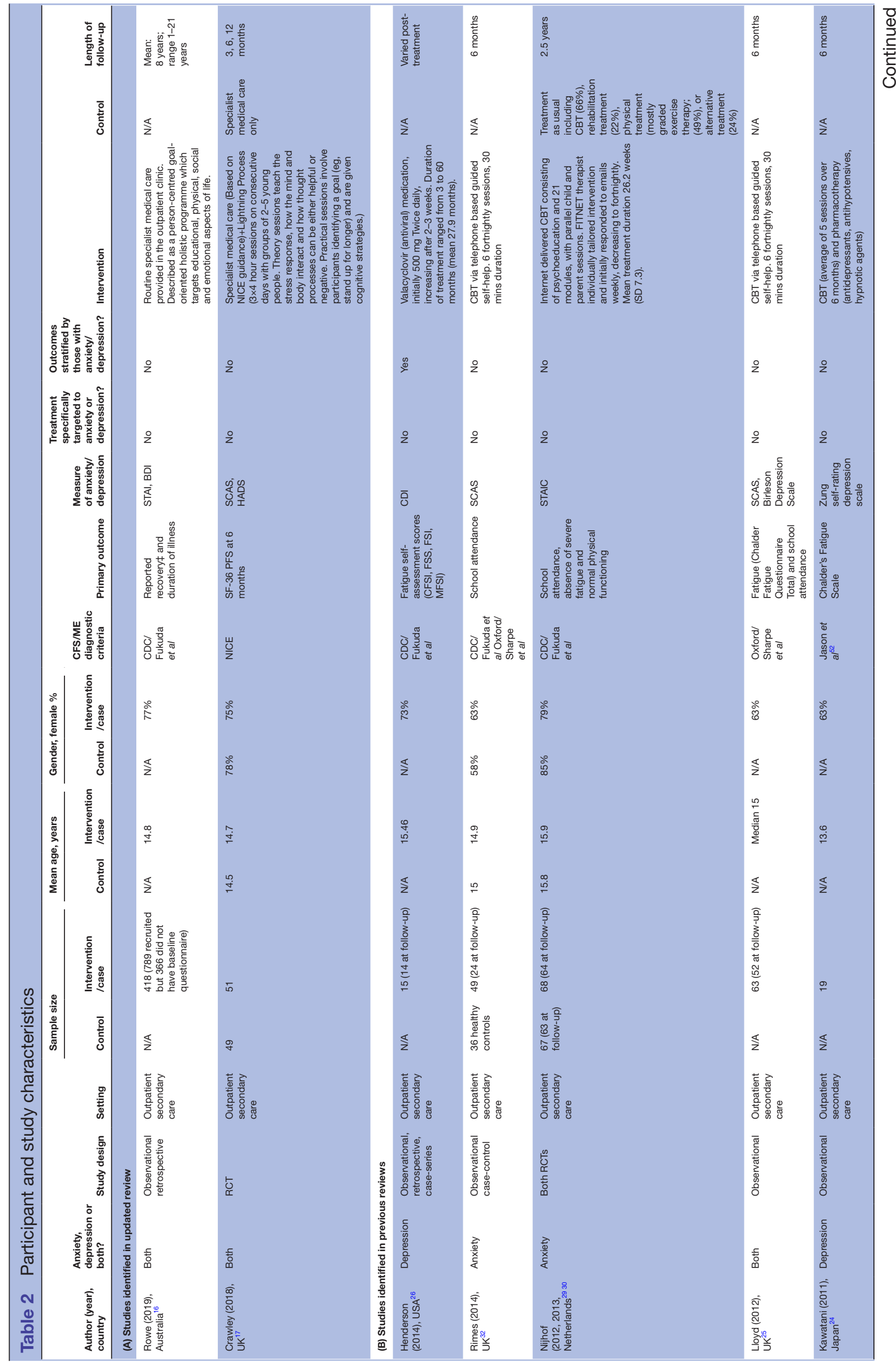




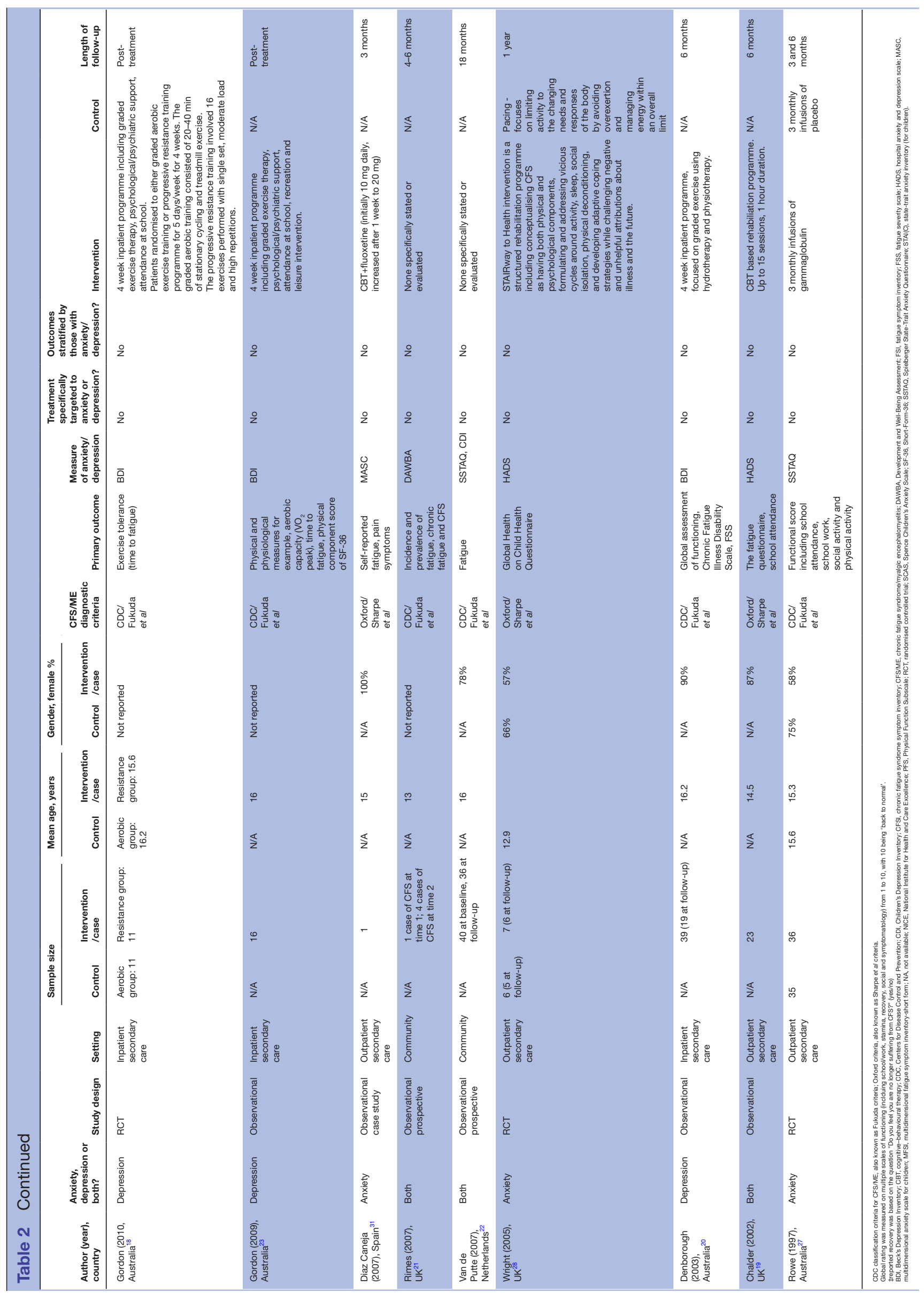

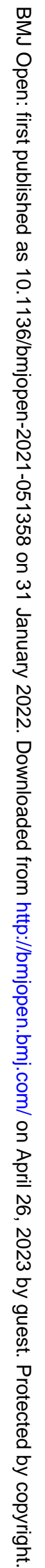


Inventory, ${ }^{39}$ the Birleson Depression Scale ${ }^{40}$ and Zung's Self-rating depression scale. ${ }^{41}$ One study used a diagnostic interview, the Development and Well-Being Assessment. ${ }^{42}$ Six studies (including the two identified in the updated review) measured both anxiety and depression; five measured depression only; and five anxiety only (table 2 ).

\section{Treatment approaches and their efficacy treating anxiety and/} or depression in paediatric CFS/ME

Of the 16 studies: one study evaluated routine specialist outpatient care $^{16}$; one evaluated the Lightening Process outpatient intervention ${ }^{17}$; one evaluated the 'STAIRway to health' outpatient intervention ${ }^{28}$; six evaluated various outpatient CBT programmes ${ }^{19} 2425$ 29-32 ; two evaluated outpatient pharmacological interventions (antivirals ${ }^{26}$ and gammaglobulins ${ }^{27}$ ); three evaluated inpatient programmes focused on graded exercise therapy ${ }^{18} 2023$; and two were epidemiological observational studies so were uninformative about interventions. ${ }^{21} 22$

There were common cognitive and behavioural elements across the behavioural and CBT programmes, including: behavioural strategies for a goal-oriented graded approach to increasing activity, often with the goal to return to full-time education or to commit to a regular activity; cognitive strategies to address the psychological implications of CFS/ME, illness-related beliefs and negative thoughts; and psychoeducation about the consequence of the illness and tools to navigate this. They varied in their intensity (eg, inpatient treatment, consecutive daily 4-hour outpatient sessions, and fortnightly 30 min phone calls), duration of treatment (days to years), and modality (eg, face to face, telephone and online). The antiviral and gammaglobuin studies did not include these elements and were distinct from the other studies in their approach.

Table 3 summarises outcomes of depression and/or anxiety and other relevant findings for each included study from (1) the updated review and (2) previous reviews. Below, we discuss the efficacy of the treatment approaches in the 14 studies which evaluated an intervention, by whether they were (1) an outpatient or (2) an inpatient programme.

\section{Outpatient programmes}

The two new studies from this updated review evaluated two outpatient programmes. Crawley et $\mathrm{al}^{17}$ compared adding the Lightening Process intervention (https:// lightningprocess.com) to specialist care (recommended by National Institute for Health and Care Excellence $\left.(\mathrm{NICE})^{1}\right)$, to specialist medical care alone. The Lightening Process is developed from osteopathy, life coaching and neurolinguistic programming and more than 250 children use it for their CFS/ME each year in the UK. ${ }^{43}$ It is delivered in intensive three, 4-hour sessions on consecutive days in small groups, with theory elements on the stress response, how the mind and body interact and how thought processes and language can be either helpful or negative, followed by practical sessions where participants identify an activity goal and are given cognitive strategies to attempt it. The study showed a significant reduction in adjusted difference in mean depressive and anxiety symptoms at 12 months $(-1.8, \mathrm{p}=0.04$ for depression; -14.5 , $\mathrm{p}<0.001$ for anxiety) among participants allocated to the Lightening Process intervention (in addition to specialist medical care) arm than those allocated to the specialist medical care-only control. The Lightening Process was more effective than specialist medical care at reducing anxiety symptoms compared with depression (at both 6 and 12 months follow-up). Outcomes in this study were not stratified by those with depression or anxiety, so we cannot comment on other CFS/ME outcomes (such as fatigue or recovery) in context of comorbid depression or anxiety.

The other study identified in this updated review evaluated routine specialist care delivered at the authors' CFS/ ME outpatient clinic in Australia. ${ }^{16}$ Routine specialist care offers a 'person-centred goal-oriented holistic programme' to 'target educational, physical, social and emotional aspects of life'. This includes symptom management (eg, sleep, migraine, dizziness, nausea, orthostatic intolerance, concentration difficulties) and focussing on increasing activity and a commitment to something enjoyable outside the home on a regular basis. This study measured depressive and anxiety symptoms at baseline but not post-treatment, so we cannot comment on the effectiveness of the intervention at reducing depression or anxiety. Instead, the study compared mean baseline depression and anxiety scores between those who had self-reported 'recovery', defined as answering 'yes' to the question 'Do you feel you are no longer suffering from CFS?' measured at a mean length of follow-up of 8 years (range 1-21). There was no difference in depression or anxiety at baseline between those who reported that they had recovered and those who had not that is, depression nor anxiety were found to be associated with recovery.

As per our previous reviews, ${ }^{11}{ }^{11}$ several studies have evaluated other outpatient programmes. Outpatient CBT interventions demonstrated inconsistent efficacy and varied in terms of delivery modality (family-focused; face to face; telephone or internet-delivered modules with therapist e-consults), intensity ( 15 weekly, hourly therapist-led sessions; six fortnightly 30 min telephone calls), duration of treatment (12 weeks to 1 year), and whether pharmacotherapy was offered alongside CBT (antidepressants and antihypotensives). Three observational studies showed that face-to-face and telephone CBT resulted in improved depression, anxiety, functioning and social adjustment. ${ }^{192532}$ An RCT showed that participants who received internet-based CBT demonstrated improvement in fatigue and school attendance at 6 months follow-up, compared with participants who received usual care ${ }^{30}$ However, the study did not measure anxiety at follow-up. Two studies that evaluated CBT alongside pharmacotherapy were uninformative as they either did not reassess mood at follow-up, ${ }^{24}$ or reported on only a single case-study. ${ }^{31}$ In terms of behavioural approaches, 


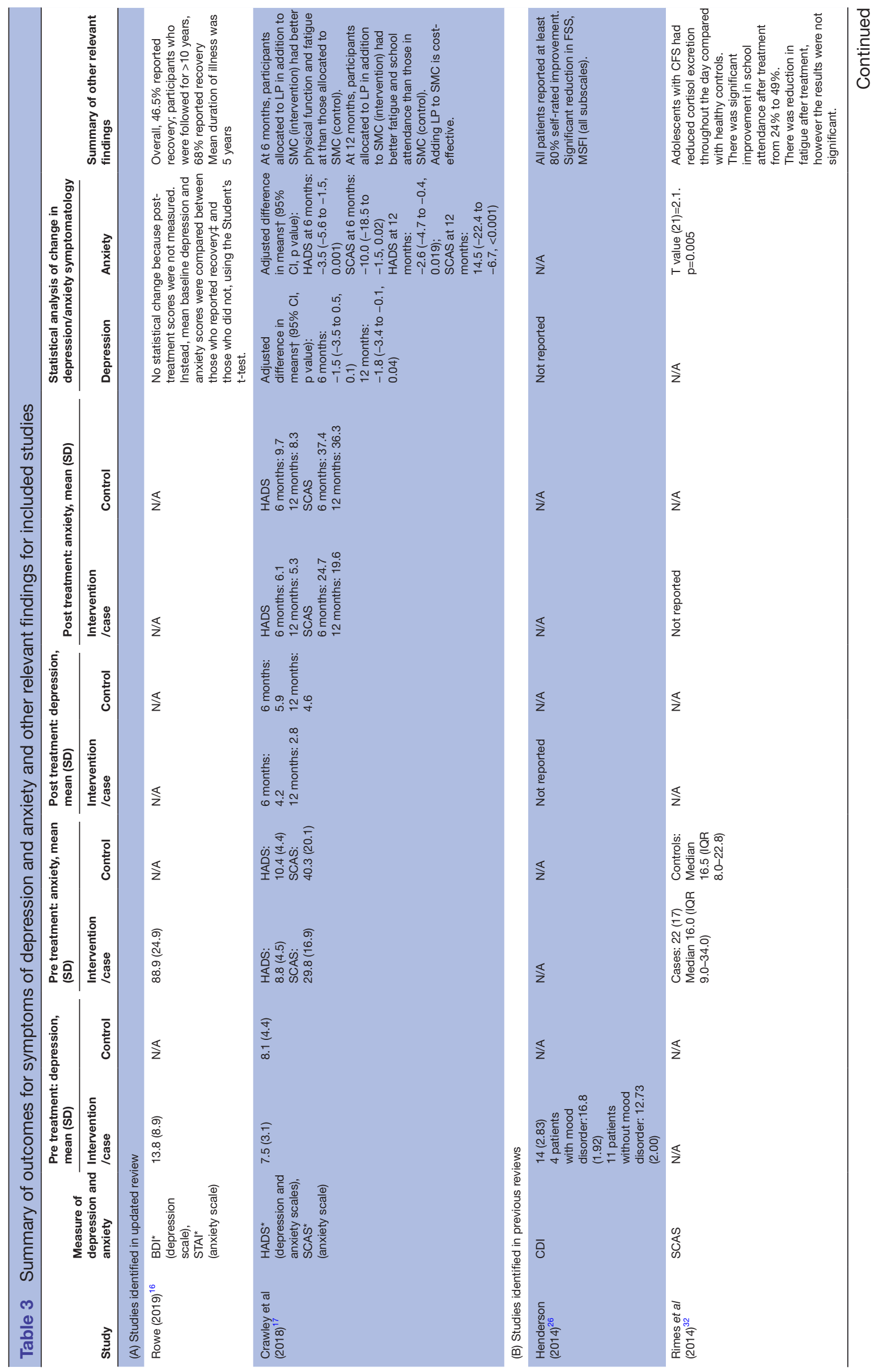




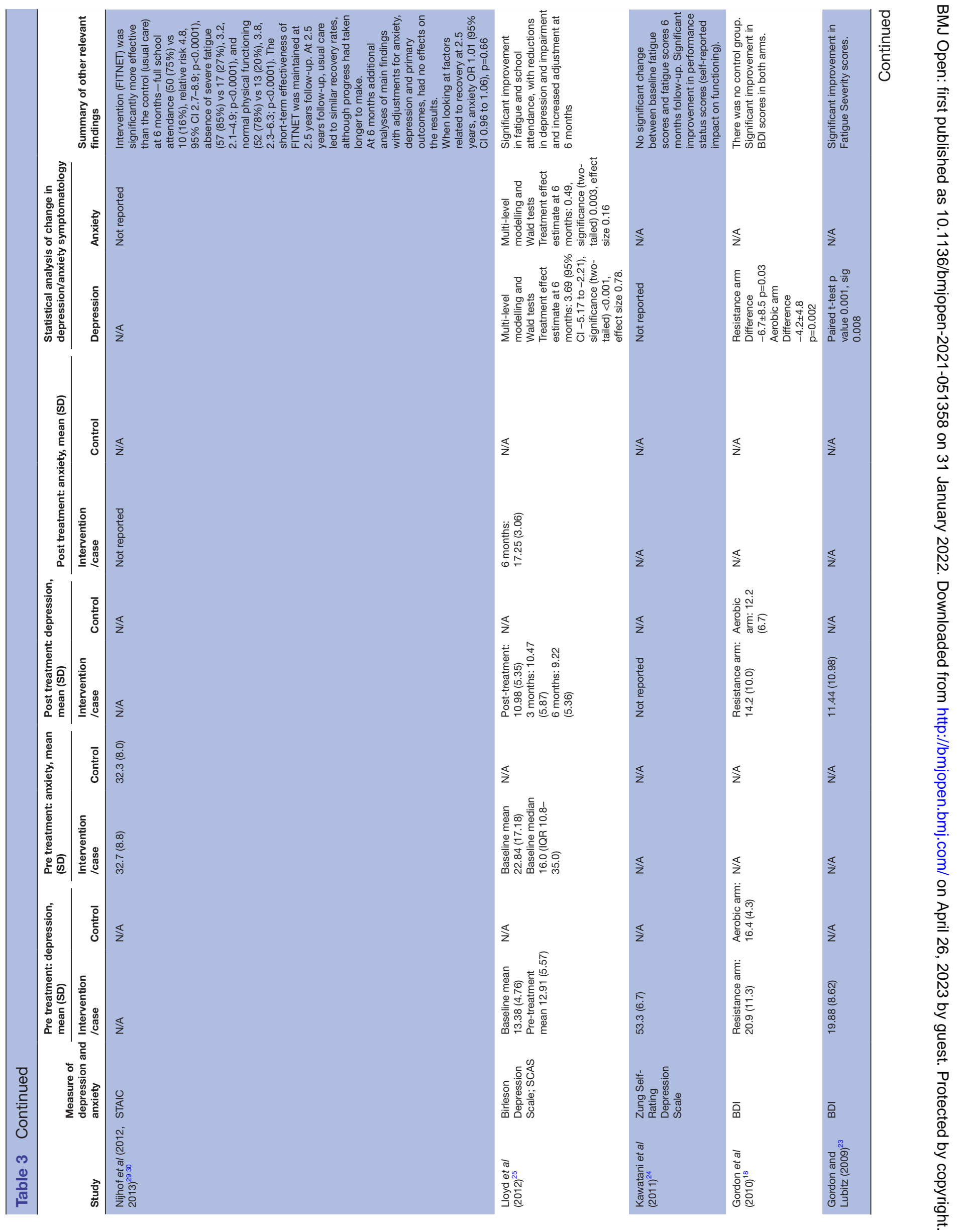




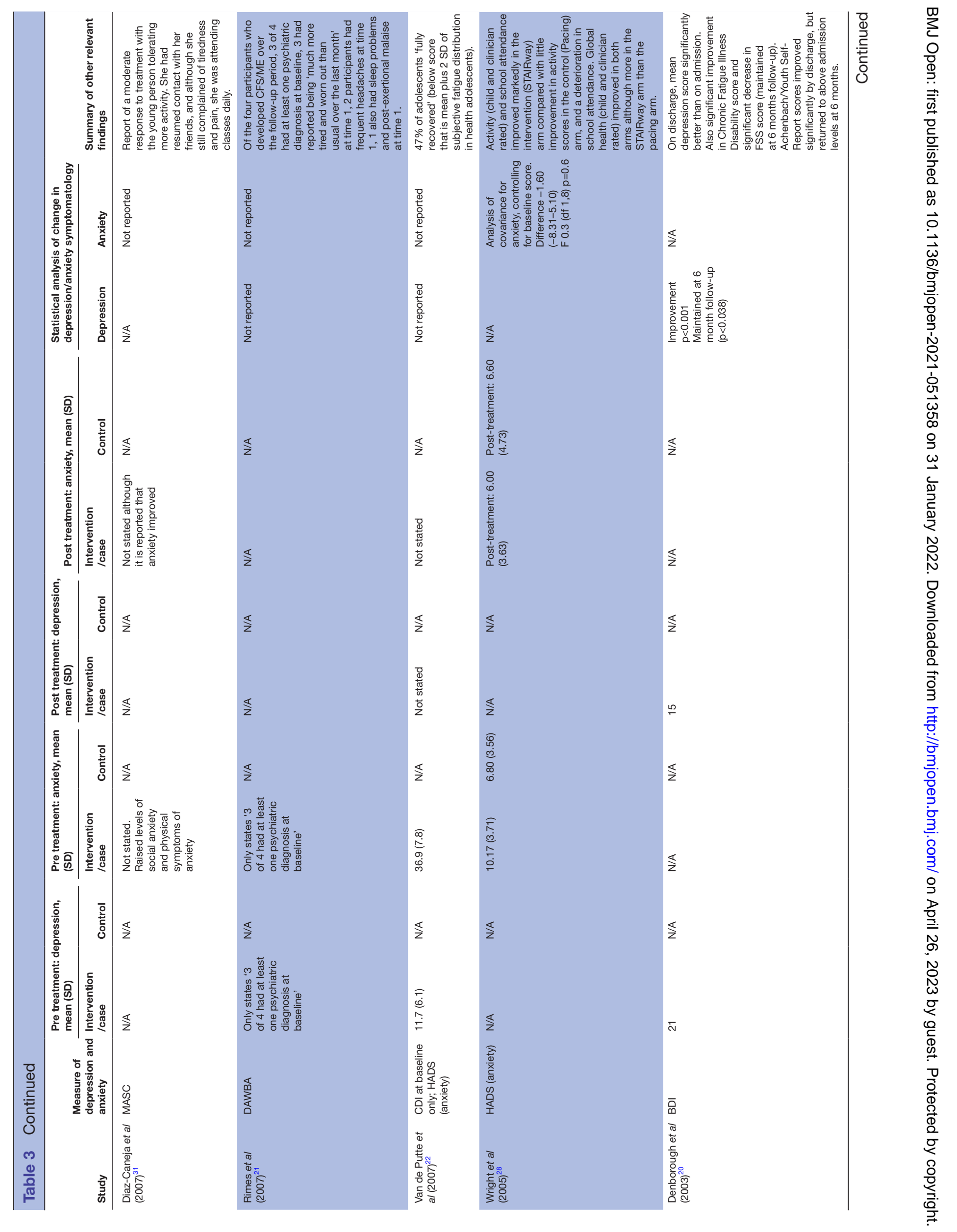




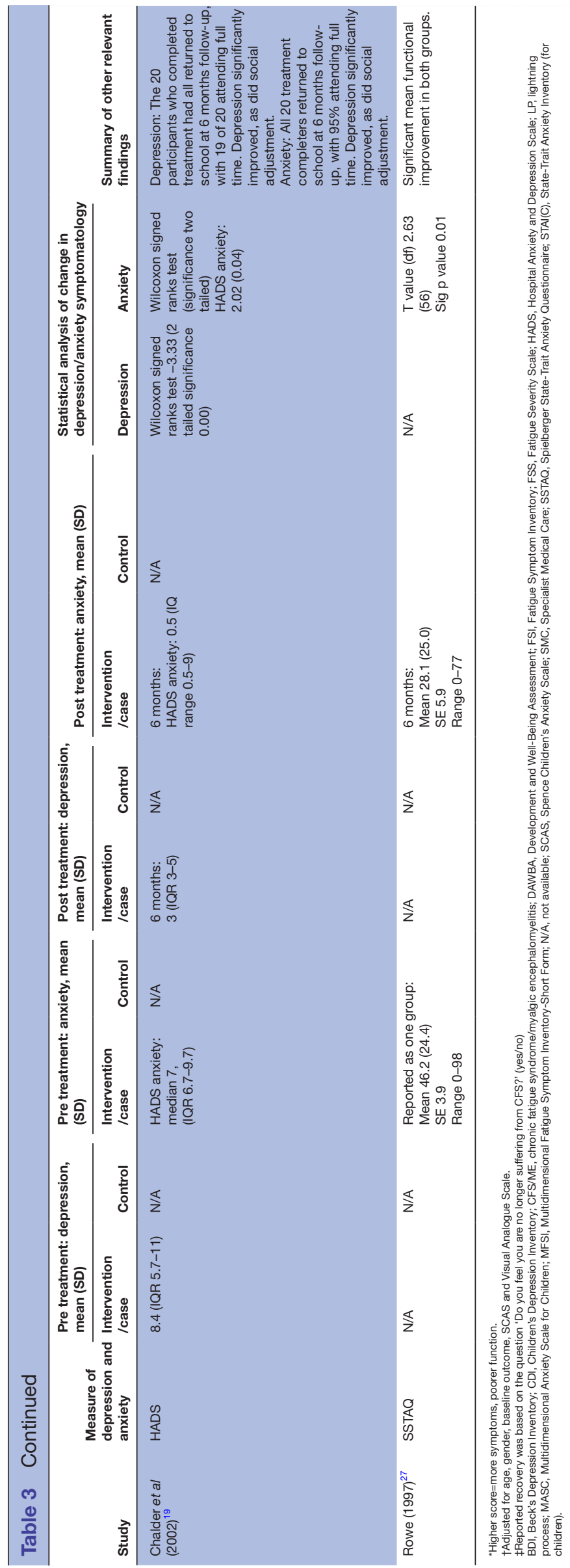


the STAIRway to Health-an incremental rehabilitation intervention-showed greater improvement in anxiety levels, when compared with a 'pacing' intervention in an RCT. $^{28}$ Pharmacological studies showed insufficient evidence for improving anxiety or depressive symptoms with intravenous gammaglobulin infusions or vancyclovir respectively ${ }^{2627}$

\section{Inpatient programmes}

As per our previous review, ${ }^{10}$ three studies ${ }^{18} 2023$ including one RCT, evidenced an improvement in mood posttreatment with a 4-week inpatient behavioural programme focused on graded exercise (including physiotherapy, aerobic exercise and resistance training), which were maintained at 6 month follow-up in one study ${ }^{20}$ ). However, they did not measure anxiety symptoms; internalising problems at 6 months returned to preadmission levels; two studies did not have follow-up data ${ }^{1823}$; all studies had small sample sizes; and the multicomponent intervention also included psychological therapy (with no further specified details about this). Therefore, these studies are uninformative for drawing conclusions about the efficacy of this behavioural intervention, or about what the key effective components of the approach may have been.

\section{DISCUSSION}

Our updated review of interventions for comorbid depression and/or anxiety in children with CFS/ME identified only two new studies published since 2015 (one of which was conducted by members of our own research team) exposing the lack of progress in this field. One study (an RCT) showed that adding the Lightening Process intervention to specialist medical care was more effective than specialist medical care alone at reducing both depressive and, to a greater extent, anxiety symptoms. The other study (an observational cohort evaluating routine specialist care) did not measure depression or anxiety at follow-up. Combined with our results from previous reviews, we identified 16 studies of 11 different interventions for paediatric CFS/ME since 1991 that include measures of anxiety and/or depression. Of these, six did not provide follow-up measurements of anxiety and/or depression post-intervention, and none of the interventions in the studies specifically targeted comorbid anxiety and/or depression. The results of this updated review do not appreciably alter what is already known from previous reviews, that there is insufficient evidence to conclude what the best interventions are for treating anxiety and/ or depression in paediatric CFS/ME patients.

Strengths of the updated review include the systematic approach, the use of four reviewers, contacting authors for subgroup data, and not limiting results to English language. The limitations are the lack of eligible studies and insufficient data available for a meta-analysis. Only two papers were eligible for inclusion, of which one did not provide sufficient follow-up data to comment on the treatment efficacy of the intervention on depression and anxiety. Neither intervention was specifically designed to measure the impact on depression and anxiety and therefore studies were inadequately powered to measure this. Studies were not stratified by those who met criteria for clinical diagnoses of depression/anxiety reducing our ability to analyse effectiveness. Furthermore, neither study used diagnostic interviews for anxiety and depression, relying instead on questionnaires. While HADS, ${ }^{44}$ SCAS $^{45}$ and STAI ${ }^{35}$ questionnaires are validated for use in adolescents, only the RCADS (Revised Children's Anxiety and Depression scale), which is derived from the SCAS, has been found to have sufficient discriminative accuracy against gold standard diagnostic interviews in paediatric CFS/ME populations. ${ }^{5}$

In conjunction with our previous reviews, we show that currently the interventions with most evidence for improvement in anxiety and depressive symptoms in CFS/ME, when compared with other interventions, such as behavioural-only or pharmacological, is CBT. ${ }^{10}{ }^{11}$ The 'Lightening Process' programme, 'STAIRway to Health' intervention, and a 4-week multicomponent inpatient rehabilitation programme show promising results for improving anxiety and/or depressive symptoms in single RCTs, but sample sizes are small and results have not been replicated. The mechanisms for why CBT could be effective are unclear because no study targeted anxiety and depression. Further, multicomponent outpatient and inpatient interventions make it difficult to identify the effective element of interventions. Our updated review does not further this debate because, while CBT is an element of 'specialist medical care' and 'routine specialist care' interventions in the new studies, we do not know how many participants received CBT or how it was delivered. Additionally, results are not stratified by those with anxiety and/or depression. Furthermore, the differences and similarities between the Lightening Process and CBT are also unclear. ${ }^{46}$ It should also be noted that the draft NICE guideline (expected publication date August 2021: https://www.nice.org.uk/guidance/gid-ng10091/documents/draft-guideline) does not recommend the Lightning Process for management of CFS (although this is not specifically aimed at anxiety and depression).

Other cognitive and behavioural based approaches are being trialled in CFS/ME, but are limited in contributing to our understanding of their efficacy for anxiety and depressive symptoms in CFS/ME because of a failure to include paediatric CFS/ME populations or those diagnosed with CFS/ME using recognised criteria, or measure anxiety and depressive symptoms in the $20 \%-30 \%{ }^{56}$ of children that experience them. Three studies ${ }^{47-49}$ were excluded from our review for these reasons. For example, studies evaluating acceptance and commitment therapy ${ }^{47}$ and mindfulness-based therapies ${ }^{48}$ show promising results in improving the physical health, symptom burden and 'emotional distress' in children with functional somatic syndromes including CFS/ME but were excluded from this review because data for adolescent participants with $\mathrm{CFS} / \mathrm{ME}$ were aggregated with those with other somatic 
syndromes, and the studies only measured general wellbeing outcomes rather than specifically validated anxiety and/or depression outcomes.

There is a pressing need for more work in this area to identify efficacious treatments for anxiety and depressive symptoms in paediatric CFS/ME so they can be used in clinical practice. We call on researchers to undertake paediatric CFS/ME interventions studies and use validated, diagnostic outcome measures of anxiety and depression.

\section{CONCLUSION}

This updated review highlights both the paucity of intervention studies in children with CFS/ME since 1991 and the lack of forward movement in identifying effective treatments for paediatric CFS/ME and comorbid depression and anxiety over the last five years. The overall quality of the literature remains poor and calls for paediatric CFS/ME intervention studies to target anxiety and depression, measure outcomes with validated scales, or report outcomes in subsets of patients with clinical diagnoses of anxiety and depression, have not been met. Given that comorbid anxiety and depression in paediatric CFS/ $\mathrm{ME}$ are associated with worse outcomes, unlikely to remit spontaneously without treatment, and can be incompatible with following standard CFS/ME treatment guidance, this needs to be addressed. Future research should improve the quality of the literature by using validated scales (as well as analyse correlation between scales) and measure anxiety and/or depression as primary outcomes in large intervention studies of comorbid anxiety and/or depression in paediatric CFS/ME.

\section{Twitter Philippa Clery @PhilippaClery}

Acknowledgements We would like to acknowledge the support from the CFS/ME teams at the Centre for Academic Child Health at the University of Bristol and the CFS/ME service at the Royal United Hospitals Bath NHS Foundation Trust.

Contributors ML and EC (guarantor) conceptualised this study. PC, AR, KD and JB performed data collection, synthesis and interpretation. PC wrote the manuscript. All authors contributed to manuscript revisions, have read the final manuscript and approved it for publication. All authors agree to be accountable for all aspects of the work.

Funding ML is funded by the National Institute for Health Research (NIHR Doctoral Research Fellowship, DRF-2016-09-021). This report is independent research.

Disclaimer The views expressed in this publication are those of the authors and not necessarily those of the NHS, NIHR or the Department of Health and Social Care.

Competing interests EC acts as a non-paid medical advisor for the Sussex and Kent ME society.

Patient consent for publication Not applicable.

Provenance and peer review Not commissioned; externally peer reviewed.

Data availability statement Data sharing not applicable as no datasets generated and/or analysed for this study. Data sharing not applicable as no datasets were generated or analysed for this study.

Supplemental material This content has been supplied by the author(s). It has not been vetted by BMJ Publishing Group Limited (BMJ) and may not have been peer-reviewed. Any opinions or recommendations discussed are solely those of the author(s) and are not endorsed by BMJ. BMJ disclaims all liability and responsibility arising from any reliance placed on the content. Where the content includes any translated material, BMJ does not warrant the accuracy and reliability of the translations (including but not limited to local regulations, clinical guidelines, terminology, drug names and drug dosages), and is not responsible for any error and/or omissions arising from translation and adaptation or otherwise.

Open access This is an open access article distributed in accordance with the Creative Commons Attribution 4.0 Unported (CC BY 4.0) license, which permits others to copy, redistribute, remix, transform and build upon this work for any purpose, provided the original work is properly cited, a link to the licence is given, and indication of whether changes were made. See: https://creativecommons.org/ licenses/by/4.0/.

ORCID iD

Philippa Clery http://orcid.org/0000-0002-6770-4454

\section{REFERENCES}

1 NICE. Chronic fatigue syndrome/myalgic encephalomyelitis (or encephalopathy): diagnosis and management (CG53), 2007. Available: https://www.nice.org.uk/guidance/CG53 [Accessed Feb 2020].

2 Lim E-J, Ahn Y-C, Jang E-S, et al. Systematic review and metaanalysis of the prevalence of chronic fatigue syndrome/myalgic encephalomyelitis (CFS/ME). J Trans/ Med 2020;18.

3 Crawley E, Sterne JAC. Association between school absence and physical function in paediatric chronic fatigue syndrome/myalgic encephalopathy. Arch Dis Child 2009;94:752-6.

4 Falk Hvidberg M, Brinth LS, Olesen AV, et al. The health-related quality of life for patients with myalgic encephalomyelitis / chronic fatigue syndrome (ME/CFS). PLoS One 2015;10:e0132421.

5 Loades ME, Read R, Smith L, et al. How common are depression and anxiety in adolescents with chronic fatigue syndrome (CFS) and how should we screen for these mental health co-morbidities? a clinical cohort study. Eur Child Adolesc Psychiatry 2021;30:1733-43.

6 Loades ME, Rimes KA, Ali S, et al. The presence of co-morbid mental health problems in a cohort of adolescents with chronic fatigue syndrome. Clin Child Psychol Psychiatry 2018;23:398-408.

7 Sadler K, Vizard T, Ford T. Mental health of children and young people in England, 2017: trends and characteristics. Available: https://digital.nhs.uk/data-and-information/publications/statistical/ mental-health-of-children-and-young-people-in-england/2017/2017 [Accessed Feb 2020].

8 Bould H, Collin SM, Lewis G, et al. Depression in paediatric chronic fatigue syndrome. Arch Dis Child 2013;98:425-8.

9 Loades ME, Rimes KA, Ali S, et al. Depressive symptoms in adolescents with chronic fatigue syndrome (CFS): are rates higher than in controls and do depressive symptoms affect outcome? Clin Child Psychol Psychiatry 2019;24:580-92.

10 Loades ME, Sheils EA, Crawley E. Treatment for paediatric chronic fatigue syndrome or myalgic encephalomyelitis (CFS/ ME) and comorbid depression: a systematic review. BMJ Open 2016;6:e012271.

11 Stoll SVE, Crawley E, Richards V, et al. What treatments work for anxiety in children with chronic fatigue syndrome/myalgic encephalomyelitis (CFS/ME)? systematic review. BMJ Open 2017;7:e015481.

12 Moher D, Liberati A, Tetzlaff J. Preferred reporting items for systematic reviews and meta-analyses: the PRISMA statement. PLoS Med 2009;6.

13 Sterne JA, Hernán MA, Reeves BC, et al. ROBINS-I: a tool for assessing risk of bias in non-randomised studies of interventions. BMJ 2016;355:i4919.

14 Sterne JAC, Savović J, Page MJ, et al. RoB 2: a revised tool for assessing risk of bias in randomised trials. BMJ 2019;366:14898.

15 Campbell M, McKenzie JE, Sowden A, et al. Synthesis without meta-analysis (swim) in systematic reviews: reporting guideline. BMJ 2020;368:16890.

16 Rowe KS. Long term follow up of young people with chronic fatigue syndrome attending a pediatric outpatient service. Front Pediatr 2019;7:21.

17 Crawley EM, Gaunt DM, Garfield K, et al. Clinical and costeffectiveness of the lightning process in addition to specialist medical care for paediatric chronic fatigue syndrome: randomised controlled trial. Arch Dis Child 2018;103:155-64.

18 Gordon BA, Knapman LM, Lubitz L. Graduated exercise training and progressive resistance training in adolescents with chronic fatigue syndrome: a randomized controlled pilot study. Clin Rehabil 2010;24:1072-9.

19 Chalder T, Tong J, Deary V. Family cognitive behaviour therapy for chronic fatigue syndrome: an uncontrolled study. Arch Dis Child 2002;86:95-7. 
20 Denborough P, Kinsella S, Stevens J, et al. Evaluation of a multidisciplinary inpatient rehabilitation programme for adolescents with chronic fatigue syndrome. Australas Psychiatry 2003;11:319-24

21 Rimes KA, Goodman R, Hotopf $M$, et al. Incidence, prognosis, and risk factors for fatigue and chronic fatigue syndrome in adolescents: a prospective community study. Pediatrics 2007;119:e603.

22 van de Putte EM, Engelbert RHH, Kuis W, et al. Alexithymia in adolescents with chronic fatigue syndrome. J Psychosom Res 2007;63:377-80.

23 Gordon B, Lubitz L. Promising outcomes of an adolescent chronic fatigue syndrome inpatient programme. J Paediatr Child Health 2009;45:286-90.

24 Kawatani J, Mizuno K, Shiraishi S, et al. Cognitive dysfunction and mental fatigue in childhood chronic fatigue syndrome--a 6-month follow-up study. Brain Dev 2011;33:832-41.

25 Lloyd S, Chalder T, Sallis HM, et al. Telephone-based guided self-help for adolescents with chronic fatigue syndrome: a nonrandomised cohort study. Behav Res Ther 2012;50:304-12.

26 Henderson TA. Valacyclovir treatment of chronic fatigue in adolescents. Adv Mind Body Med 2014;28:4-14

27 Rowe KS. Double-blind randomized controlled trial to assess the efficacy of intravenous gammaglobulin for the management of chronic fatigue syndrome in adolescents. J Psychiatr Res 1997;31:133-47.

28 Wright B, Ashby B, Beverley D, et al. A feasibility study comparing two treatment approaches for chronic fatigue syndrome in adolescents. Arch Dis Child 2005;90:369-72.

29 Nijhof SL, Bleijenberg G, Uiterwaal CSPM, et al. Effectiveness of Internet-based cognitive behavioural treatment for adolescents with chronic fatigue syndrome (FITNET): a randomised controlled trial. Lancet 2012;379:1412-8.

30 Nijhof SL, Priesterbach LP, Uiterwaal CSPM, et al. Internet-Based therapy for adolescents with chronic fatigue syndrome: long-term follow-up. Pediatrics 2013;131:e1788-95.

31 Díaz-Caneja Greciano A, Rodríguez Sosa JT, Aguilera Albesa S, et al. [Chronic fatigue syndrome in a 15-year-old girl]. An Pediatr 2007;67:74-7.

32 Rimes KA, Papadopoulos AS, Cleare AJ, et al. Cortisol output in adolescents with chronic fatigue syndrome: pilot study on the comparison with healthy adolescents and change after cognitive behavioural guided self-help treatment. J Psychosom Res 2014;77:409-14.

33 Zigmond AS, Snaith RP. The hospital anxiety and depression scale. Acta Psychiatr Scand 1983;67:361-70.

34 Spence SH, Barrett PM, Turner CM. Psychometric properties of the Spence children's anxiety scale with young adolescents. J Anxiety Disord 2003;17:605-25.

35 Spielberger CD. Manual for the state trait anxiety inventory for children. Palo Alto: Consulting Psychologists Press, 1973.

36 March JS, Parker JD, Sullivan K, et al. The multidimensional anxiety scale for children (MASC): factor structure, reliability, and validity. $J$ Am Acad Child Adolesc Psychiatry 1997;36:554-65.
37 Spielberger C. Self-evaluation questionnaire state trait anxiety inventory. Palo Alto, CA: Consulting Psychologists Press, 1977.

38 Beck AT, Steer RA, Carbin MG. Psychometric properties of the Beck depression inventory: twenty-five years of evaluation. Clin Psychol Rev 1988;8:77-100.

39 Saylor CF, Finch AJ, Spirito A, et al. The children's depression inventory: a systematic evaluation of psychometric properties. $J$ Consult Clin Psychol 1984;52:955-67.

40 Birleson P, Hudson I, Buchanan DG, et al. Clinical evaluation of a self-rating scale for depressive disorder in childhood (depression self-rating scale). J Child Psychol Psychiatry 1987;28:43-60.

41 Zung WW. A self-rating depression scale. Arch Gen Psychiatry 1965;12:63-70.

42 Goodman R, Ford T, Richards H, et al. The development and wellbeing assessment: description and initial validation of an integrated assessment of child and adolescent psychopathology. J Child Psychol Psychiatry 2000;41:645-55.

43 Reme SE, Archer N, Chalder T. Experiences of young people who have undergone the Lightning Process to treat chronic fatigue syndrome/myalgic encephalomyelitis-a qualitative study. $\mathrm{Br} \mathrm{J}$ Health Psychol 2013;18:508-25.

44 White D, Leach C, Sims R, et al. Validation of the hospital anxiety and depression scale for use with adolescents. Br J Psychiatry 1999;175:452-4

45 Orgilés M, Fernández-Martínez I, Guillén-Riquelme A, et al. A systematic review of the factor structure and reliability of the Spence children's anxiety scale. J Affect Disord 2016;190:333-40.

46 Anderson E, Loades M, Starbuck J. CBT repackaged or a novel treatment? the lightning Process $₫$ compared with specialist medical care for paediatric chronic fatigue syndrome. Biomed Health Behav 2021:1-20.

47 Kallesøe KH, Schröder A, Wicksell RK, et al. Feasibility of groupbased acceptance and commitment therapy for adolescents (ahead) with multiple functional somatic syndromes: a pilot study. $B M C$ Psychiatry 2020;20:457

48 Ali A, Weiss TR, Dutton A, et al. Mindfulness-Based stress reduction for adolescents with functional somatic syndromes: a pilot cohort study. J Pediatr 2017;183:184-90.

49 Kluck BN, Junghans-Rutelonis AN, Jones AE. Adolescent chronic fatigue and orthostatic intolerance. Clinical Pediatrics 2017;56:85-9.

50 Fukuda K, Straus SE, Hickie I, et al. The chronic fatigue syndrome: a comprehensive approach to its definition and study. International chronic fatigue syndrome Study Group. Ann Intern Med 1994;121:953-9.

51 Sharpe MC, Archard LC, Banatvala JE, et al. A report-chronic fatigue syndrome: guidelines for research. J $R$ Soc Med 1991;84:118-21.

52 Jason LA, Jordan K, Miike T, et al. A pediatric case definition for myalgic encephalomyelitis and chronic fatigue syndrome. J Chronic Fatigue Syndr 2006;13:1-44. 